\title{
Efeito das intervenções assistidas por animais sobre o bem-estar e parâmetros fisiológicos de estudantes universitários
}

\author{
Effect of animal-assisted interventions on the well-being and physiological parameters of university
} students

Efecto de las intervenciones asistidas por animales sobre el bienestar y los parámetros fisiológicos de los estudiantes universitarios

\begin{abstract}
Resumo
O ambiente universitário pode desencadear uma piora no bem-estar dos jovens acadêmicos e muitos gestores de universidade estão buscando estratégias para esse problema, como por exemplo a intervenção assistida por animais. $\mathrm{O}$ objetivo do estudo foi avaliar o efeito da interação com cães coterapeutas sobre o bem-estar e os parâmetros fisiológicos como pulso, pressão arterial sistólica e diastólica de estudantes de dois cursos universitários em Pelotas, RS. Participaram 38 acadêmicos da área da saúde animal (Medicina Veterinária) e humana (Psicologia). Foi realizada uma intervenção para cada curso na qual os estudantes interagiram com cães coterapeutas a partir de atividades lúdicas. A avaliação ocorreu pré e pós-intervenção, sendo que o bem-estar subjetivo foi mensurado, através da Escala de Faces, apenas dos estudantes de veterinária e o pulso, a pressão arterial sistólica e a diastólica foram mensuradas apenas dos estudantes de psicologia. Os resultados foram obtidos através do teste $t$ de Student pareado. Foi observado uma melhora significativa nas expressões faciais dos estudantes ao avaliar pré $(0,78 \pm 1,043)$ e pós-intervenção com os cães coterapeutas $(2,70 \pm 0,635)$, além de reduções significativas nos valores médios pré e pós-intervenção do pulso, diminuindo de 85,13 para 80,87 bpm $(\mathrm{p}=0,025)$ e também na pressão arterial sistólica, em decréscimo de 118,67 bpm para 108,00 bpm, ( $\mathrm{p}=0,010)$. Não houve redução significativa na pressão arterial diastólica. Estes resultados permitem concluir que houve efeito positivo sobre o bem-estar e parâmetros fisiológicos dos estudantes a partir de uma única intervenção.
\end{abstract}

Palavras-chave: Cães coterapeutas; Pressão arterial; Pulso; Expressões faciais.

\section{Abstract}

The university environment can trigger a worsening in the well-being of young academics and many university managers are seeking strategies for this problem, such as animal-assisted intervention. The aim of the study was to evaluate the effect of interaction with co-therapist dogs on well-being and physiological parameters such as pulse, systolic and diastolic blood pressure of students from two university courses in Pelotas, RS. Thirty-eight university 
students from the field of animal health (Veterinary Medicine) and human health (Psychology) participated. An intervention was performed lasting 40 to 50 minutes for each in which the students interacted with four co-therapist dogs based on playful activities. The evaluations occurred before and after the intervention, and subjective well-being was measured through the Andrews Face Scale only of veterinary students and by measuring pulse, systolic and diastolic blood pressure only of psychology students. The results were obtained from the paired Student's T-test. A significant improvement was observed in the facial expressions of the students when evaluating pre $(0.78 \pm 1.043)$ and post-intervention with the co-therapist $\operatorname{dogs}(2.70 \pm 0.635)$, in addition to significant reductions in the mean values before and after pulse intervention, decreasing from 85.13 to $80.87 \mathrm{bpm}(\mathrm{p}=0.025)$ and also in systolic blood pressure, decreasing from 118.67 to $108.00 \mathrm{bpm}(\mathrm{p}=0.010)$. There was no significant reduction in diastolic blood pressure. These results allowed to conclude that there was a positive effect on the well-being and physiological parameters of the students from a single intervention.

Keywords: Co-therapist dogs; Blood pressure; Pulse; Facial expressions.

\section{Resumen}

El entorno universitario puede provocar un empeoramiento en el bienestar de los jóvenes académicos y muchos directivos universitarios están buscando estrategias para este problema, como la intervención asistida por animales. El objetivo del estudio fue evaluar el efecto de la interacción con perros coterapeutas sobre el bienestar y parámetros fisiológicos como el pulso, la presión arterial sistólica y diastólica de estudiantes de dos cursos universitarios en Pelotas, RS. Participaron 38 estudiantes de dos cursos, Medicina Veterinaria y Psicología. Para cada curso se realizó una intervención en la que los estudiantes interactuaron con perros coterapeutas a través de actividades recreativas. La evaluación se realizó antes y después de la intervención, y se midió el bienestar subjetivo utilizando la Escala de Rostro solo para estudiantes de veterinaria y se midió el pulso, presión arterial sistólica y diastólica solo para estudiantes de psicología. Los resultados se obtuvieron mediante la prueba t de Student pareada. Se observó una mejora significativa en las expresiones faciales de los estudiantes al evaluar antes $(0,78 \pm 1,043)$ y después de la intervención con perros coterapeutas $(2,70 \pm 0,635)$, además de reducciones significativas en los valores medios del pulso, disminuyendo de 85,13 a 80,87 lpm $(\mathrm{p}=0,025)$ y también en la presión arterial sistólica, de 118,67 a 108,00 lpm, $(\mathrm{p}=0,010)$. No hubo una reducción significativa de la presión diastólica. Estos resultados nos permiten concluir que hubo un efecto positivo sobre el bienestar y los parámetros fisiológicos de los estudiantes a partir de una sola intervención.

Palabras clave: Perros coterapeutas; Presión arterial; Pulso; Expresiones faciales.

\section{Introdução}

A busca pela profissão ideal e as exigências criadas pelo mercado de trabalho faz com que a formação acadêmica seja cada vez mais necessária. O ingresso na universidade traz novas descobertas, desenvolvimento pessoal e conhecimento técnico, no entanto a inserção no ambiente universitário pode desencadear prejuízos na qualidade de vida e bem-estar dos jovens (Fernandez et al., 2016; Graner \& Ramos-Cerqueira, 2019).

O bem-estar pode ser definido como avaliação subjetiva da qualidade vida das pessoas, abrangendo aspectos como a felicidade, satisfação e afeto positivo, ou simplesmente como as pessoas experienciam suas vidas de forma positiva (Diener, 1996). De acordo com o autor as definições de bem-estar podem ser categorizadas sobre três aspectos.

O primeiro aspecto considera que o bem-estar é definido em critérios externos, logo não é visto como subjetivo, e sim algo desejável. O segundo pondera sobre o porquê de as pessoas avaliarem suas vidas baseadas em termos positivos; neste caso, o indivíduo avalia o que é bem-estar de acordo com seu embasamento próprio, sendo relatado que $99 \%$ de entrevistados já fizeram este tipo de questionamento sobre suas vidas (Andrews \& Whitey, 1976). Já o último aspecto considera como o bem-estar é discorrido no cotidiano, e o prevalecimento do afeto positivo sobre afeto negativo (Bradburn,1969), ou seja, ressaltando o bem-estar como uma experiência satisfatória. (Diener, 1996).

Em relação ao bem-estar de estudantes universitários, estratégias que promovam fatores ou vivências acadêmicas positivas resultam em melhoria da qualidade de vida, principalmente quando há o envolvimento extra-acadêmico, em atividades físicas, promoção da cultura, lazer e participação de grupos de estudo, por exemplo (Oliveira, 2006; NogueiraMartins, 2018). Já deficiências na manutenção desse bem-estar pode impactar negativamente no desempenho dos estudantes, principalmente pela falta de disciplina, sobrecarga de tarefas estudantis, mal gerenciamento de tempo e estresse (Fonaprace, 
2019).

Desta forma, é imprescindível que as universidades estejam capacitadas a subsidiar além da formação acadêmica, priorizando também o desenvolvimento social, cognitivo e emocional dos estudantes (Schleich, 2006). Observando tal demanda, muitos gestores buscam estratégias para promover vivências positivas, por exemplo, implementando momentos de interação com animais coterapeutas ou Intervenções Assistidas por Animais (IAAs) (Crossman \& Kazdin, 2015).

De acordo com a International Association of Human-Animal Interaction Organizations (IAHAIO) as intervenções assistidas por animais são intervenções estruturadas e com objetivos bem definidos que inclui animais na saúde, educação ou demais atividades com finalidade terapêutica em humanos (Jegatheesan et al., 2014). É uma prática multi, inter e transdisciplinar que possibilita uma maior integração com o assistido, o animal coterapeuta e o profissional atuante, seja ele da saúde ou educação (Nobre et al., 2017). Diversos animais podem ser considerados coterapeutas, como pássaros, coelhos e cavalos, porém a inclusão de cães é feita em larga escala, principalmente por serem dóceis e de fácil treinamento (Fine, 2010).

Os benefícios gerados pelas IAAs em prol de estudantes são amplamente relatados, principalmente no âmbito psicoemocional (Bjick, 2012; Haggerty \& Mueller, 2017; Williams et al., 2018; Pendry et al., 2020). Com apenas uma sessão de IAAs, estudantes que interagiram com cães coterapeutas relataram menor saudade de casa, mais senso de pertencimento pelo campus universitário e redução no estresse percebido (Binfet, 2017). Dada a atualidade e importância do tema, o presente estudo teve por objetivo avaliar o efeito da interação com cães coterapeutas sobre o bem-estar e os parâmetros fisiológicos como pulso, pressão arterial sistólica e diastólica de estudantes universitários.

\section{Metodologia}

\section{Participantes}

O presente estudo de caráter quanti-qualitativo (Estrela, 2018) teve como amostra 38 acadêmicos da área da saúde animal (Medicina Veterinária) e humana (Psicologia), abrangendo respectivamente alunos do primeiro e décimo semestre. Os estudantes foram recrutados voluntariamente e preencheram o Termo de Consentimento Livre e Esclarecido para participarem da atividade.

\section{Cães}

Foram selecionados quatro cães coterapeutas do Pet Terapia, projeto de pesquisa, ensino e extensão associado a Faculdade de Veterinária da Universidade Federal de Pelotas, que atua em Intervenções Assistidas por Animais há 14 anos. Os cães eram castrados, sem raça definida, adultos e diferentes sexos, de porte médio a grande e temperamento ativo e alegre. O treinamento e capacitação, a fim de que o cão esteja apto a atuar como coterapeuta, é realizado pela equipe do projeto, sendo nesses contemplados técnicas de adestramento, socialização com pessoas e animais, além de dessensibilização tátil, sonora e desenvolvimento de habilidades inatas e em jogos interativos. Durante as intervenções, os cães foram conduzidos por colaboradores do projeto e o comportamento e nível de bem-estar foram avaliados por médicos veterinários especialistas em intervenções assistidas por animais.

A saúde e higiene dos cães foi garantida através de um protocolo higiênico-sanitário que abrange escovação dentária diária, banhos com shampoo neutro no dia da intervenção, corte de unha, tosa higiênica, vacinação anual e controle parasitário. Além disso, são realizados periodicamente exames clínicos e de rotina (hematológicos e bioquímicos, coproparasitológico e de imagem), priorizando a segurança no contato com os animais coterapeutas. 


\section{Instrumentos}

Os 15 estudantes de psicologia tiveram Pressão Arterial Sistólica (PAS), Diastólica (PAD) e pulso mensurados por meio de um medidor de pressão digital Techline Z-46. Já os 23 estudantes de Medicina Veterinária somente responderam a Escala de Faces de Andrews (Mcdowell, 2006), adaptada para avaliar o nível de bem-estar ao interagir com os cães. A Escala de Faces é uma escala visual que contempla sete expressões faciais organizadas de A ao G. As faces são representadas por um círculo com olhos que não se alteram e sorrisos que variam de um semicírculo voltado pra cima representando muito feliz (A) a um semicírculo semelhante voltado pra baixo representando muito triste $(\mathrm{G})$ e foi adaptada para medir o grau de bem-estar, atribuindo números decrescentes de acordo com as expressões faciais de A a F, em que A condiz a 3, B a 2, C a 1, D a 0, E a 1, F a -2 e G a -3. Todos os instrumentos foram aplicados pré e pós-intervenção.

\section{Procedimentos}

O procedimento foi realizado igualmente, porém em dias diferentes para os estudantes de medicina veterinária e para os estudantes de psicologia e teve a duração de 40 a 50 minutos. Os participantes foram convidados a entrar numa sala previamente organizada dispondo de cadeiras e espaço amplo e foi explicado brevemente o procedimento bem como apresentado os cães coterapeutas que participariam.

Foi realizada uma dinâmica em que os cães estavam vestidos com um colete de bolsos contendo papéis com instruções de atividades para os participantes realizarem (por exemplo, dançar, cantar, abraçar) com o intuito de reduzir a timidez e expandir a comunicação entre eles. Seis participantes escolhiam um cão, retirava o papel do colete e executava a atividade junto ao grupo.

A partir disso, os participantes foram separados aleatoriamente em quatro grupos identificados por cor e pela escolha do cão coterapeuta que mais os cativaram. Foram desenvolvidas três atividades lúdicas de interação direta com o cão coterapeuta. Na primeira, cada grupo resolveu um quebra-cabeça em formato de casa com seis formas geométricas enquanto faziam carinho e interagiam com o cão coterapeuta cuja. Essa atividade teve o intuito de estimular raciocínio lógico e trabalho em equipe.

Na segunda atividade nomeada 'corrida do ovo', cada participante passou por um circuito segurando com a boca uma colher descartável com uma bola de ping-pong. Durante o percurso com obstáculos, os cães estavam em pontos estratégicos e os participantes deveriam fazer carinho neles; ao final do circuito outro participante pegava a bola, colocava em outra colher descartável e iniciava o circuito novamente. Essa atividade teve o intuito estimular o equilíbrio e coordenação motora.

$\mathrm{Na}$ terceira atividade nomeada "passa-anel", os participantes se sentaram formando um círculo e duas pessoas escondiam um petisco entre a palma das mãos. Com a ajuda do condutor, outro participante conduzia o cão coterapeuta a fim de que este encontrasse o petisco. O cão do grupo que achasse o petisco primeiro ganhava a brincadeira. Essa atividade teve o intuito de promover foco, atenção e socialização.

Ao final das três atividades os participantes se despediam dos cães e forneciam um feedback das emoções percebidas na intervenção por meio da escrita em superfície de balões. Os participantes escreviam no balão, jogavam-no para cima, recolhia-o aleatoriamente, liam a mensagem para o grupo e estourava o balão que continham em seu interior um papel com frases de motivação e agradecimento pela participação.

\section{Análise de dados}

Os dados foram coletados durante o segundo semestre de 2019. A análise estatística foi processada pelo software IBM SPSS 20 (IBM Corp, Armonk, New York) e os resultados obtidos a partir do Teste t de Student pareado, utilizado para determinar as diferenças pré e pós-intervenção. A significância assumida neste estudo foi de p <0,05. 


\section{Considerações éticas}

A intervenção foi elaborada sob as normas do Comitê de Ética em Pesquisa da Faculdade de Medicina (nº 3.977.986) e submetido à apreciação da Comissão de Ética em Experimentação Animal da Faculdade de Medicina Veterinária (nº 9431), sendo aprovado em ambas as instâncias da Universidade Federal de Pelotas.

\section{Resultados e Discussão}

Foi observada uma melhora significativa $(\mathrm{p}=0,00)$ nas expressões faciais dos estudantes ao avaliar pré $(0,78 \pm 1,043)$ e pós-intervenção com os cães coterapeutas $(2,70 \pm 0,635)$, com uma diferença média de $-1,913$. Este resultado também é visualmente percebido (Figura 1) pelo aumento da quantidade de expressão facial A, em que pré-intervenção não havia sido assinalada e pós-intervenção passou a dezoito. O mesmo efeito acontece nas quantidades das expressões faciais $\mathrm{D}$ e $\mathrm{F}$ que respectivamente passaram de seis e três respostas a zero respostas pós-intervenção.

Figura 1. Histograma sobre a escala de Faces de Andrews e respostas obtidas dos estudantes de medicina veterinária pré e pósintervenção com os cães coterapeutas.

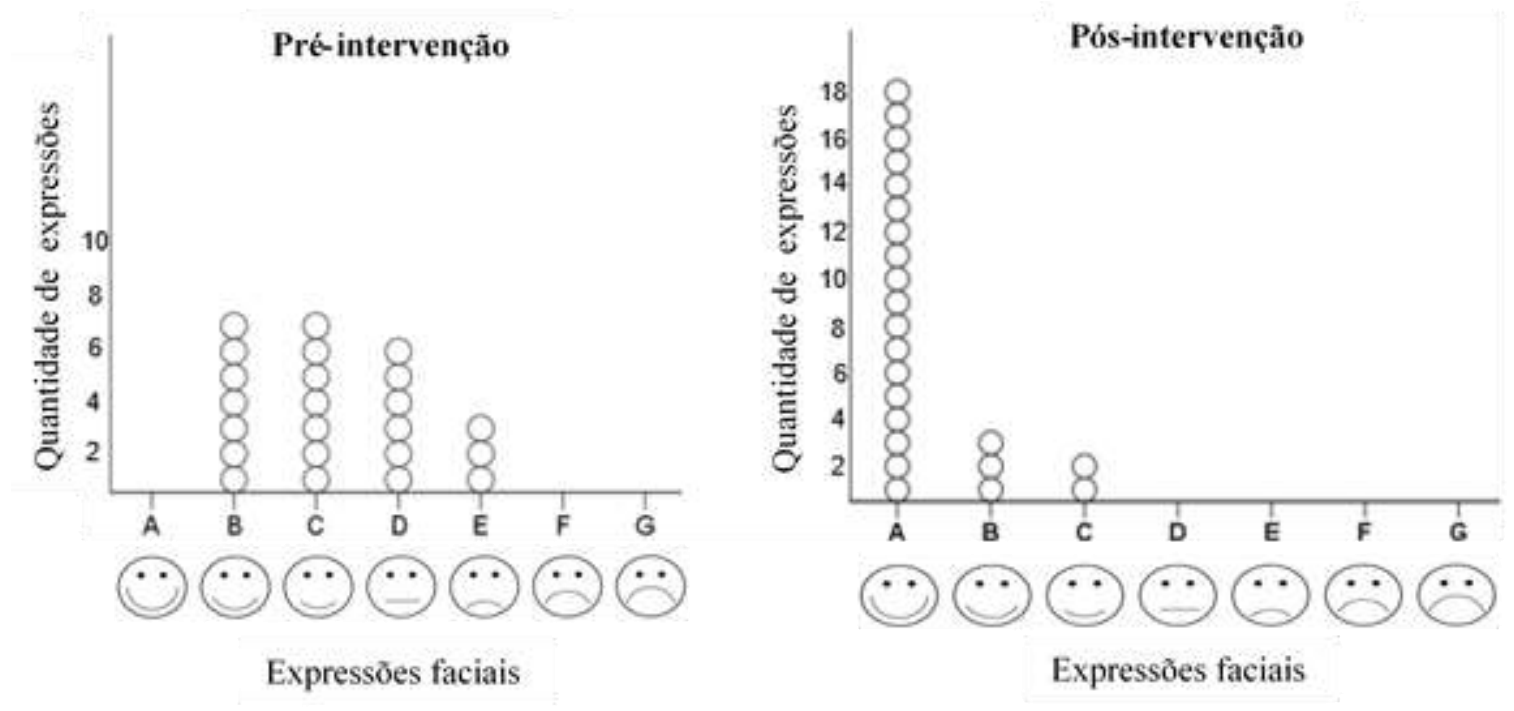

Fonte: Autores.

O eixo y refere-se a quantidade de expressões e o eixo x refere-se ao tipo de expressão, sendo estas classificadas de A a G, respectivamente do muito feliz ao muito triste. Fonte: Arquivo pessoal dos autores.

Os parâmetros fisiológicos compreendidos neste estudo pelo pulso, pressão arterial sistólica e diastólica estão descritos na Tabela 1. Foram observadas reduções significativas nos valores médios pré e pós-intervenção do pulso, diminuindo de 85,13 para 80,87 bpm, com uma diferença média de 4,267 bpm ( $\mathrm{p}=0,025)$ e também na pressão arterial sistólica, em decréscimo de 118,67 bpm para 108,00 bpm, com uma diferença média de 10,667 bpm (p = 0,010). Não houve redução significativa na pressão arterial diastólica $(\mathrm{p}=0,818)$. 
Tabela 1. Análise estatística da mensuração de pulso, pressão arterial sistólica e diastólica dos estudantes de Psicologia pré e pós-intervenção com cães coterapeutas.

\begin{tabular}{lcccccc} 
& Pré-intervenção & Pós-intervenção & & & \\
\cline { 2 - 3 } & $\mathrm{M} \pm \mathrm{DP}$ & $\mathrm{M} \pm \mathrm{DP}$ & & Diferença Média $\pm \mathrm{DP}$ & $\mathrm{t}$ \\
\hline Pulso* $^{*}(\mathrm{bpm})$ & $85,13 \pm 8,741$ & $80,87 \pm 10,901$ & & $4,267 \pm 6,606$ & 2,502 \\
PAS** $(\mathrm{mmHg})$ & $118,67 \pm 12,459$ & $108,00 \pm 17,809$ & & $10,667 \pm 13,870$ & 2,978 \\
PAD $(\mathrm{mmHg})$ & $74,67 \pm 9,904$ & $74,00 \pm 9,856$ & & $0,667 \pm 10,998$ & 0,235 \\
\hline
\end{tabular}

$* \mathrm{p}=0,025 ; * * \mathrm{p}=0,01 ; \mathrm{M}=$ média; $\mathrm{DP}=$ desvio-padrão; $\mathrm{PAS}=$ pressão arterial sistólica; $\mathrm{PAD}=$ pressão arterial diastólica; bpm = batimentos por minuto. $\mathrm{mmHg}=$ milímetros de mercúrio. Teste $\mathrm{t}$ de Student pareado. Significância assumida de $\mathrm{p}<0,05$. Fonte: Autores.

O presente estudo demonstrou de fato que a interação com cães coterapeutas implica em efeitos positivos sobre o bem-estar e parâmetros fisiológicos das pessoas. Os resultados indicaram que, a partir de uma única sessão, houve um aumento das expressões positivas obtidas através de uma escala visual. Os achados corroboram com um estudo que abrange o efeito de uma curta intervenção assistida por cães com 132 estudantes universitários selecionados voluntariamente, relatando melhorias no bem-estar e humor destes (Grajfoner et al., 2017).

A escala de faces demonstrou de forma simples e rápida o estado emocional dos participantes deste estudo antes e após as IAAs. Assim como no estudo de Lussi e colaboradores (2004), a Escala de faces foi adaptada para medir o grau de bem-estar, atribuindo números decrescentes de acordo com as expressões faciais de A a F. Além de melhorias sobre o bemestar, a aplicação de IAAs pode reduzir a ansiedade e estresse proveniente do ambiente universitário (Trammel, 2017; Wood et al., 2018; Silas et al., 2019). Resultados semelhantes aos nossos já foram descritos em pessoas com transtornos mentais, a partir da interação lúdica com cães coterapeutas, indicando, desta forma, que os benefícios das IAAs podem ser vistos em diversas situações e públicos (Sapin et al., 2020).

Houve implicações positivas sobre o pulso a partir da interação dos cães com os estudantes, acarretando em redução do parâmetro pós-intervenção. Esse achado vem de encontro ao estudo desenvolvido com 48 estudantes durante a semana de exames finais, também obtendo redução do pulso pós-intervenção assistida com cães (Delgado et al., 2017).

Ao considerar a pressão arterial sistólica e diastólica, apenas a primeira teve redução significativa $(p=0,01)$. Os achados neste estudo só também corroboram com Delgado e colaboradores (2017), no entanto, a literatura difere bastante em relação a esse parâmetro. Por exemplo, os dados obtidos por Wood e colaboradores (2018) indicam um aumento significativo da pressão arterial sistólica e diastólica após uma IAA em grupos contendo seis estudantes e com duração de 15 minutos. O autor ressalva que no momento pré-intervenção, $24 \%$ dos estudantes estavam com a pressão arterial sistólica acima de 140mmHg, logo classificados em condição de hipertensão arterial leve, ou seja, com valores sistólicos entre 140 a 159 mmHg (Kohlmann Junior et al., 1999). No momento pós-intervenção o valor foi de $20 \%$. Em comparação, o presente estudo teve 13,33\% dos estudantes sob condição hipertensa leve no momento pré-intervenção e 6,66\% pós-intervenção.

Durante a primeira e segunda atividade lúdica descritas na metodologia, foi percebido que os participantes por vezes desfocavam de realizar o que era proposto e preferiam interagir diretamente com os cães, comprometendo no desempenho do grupo e no ganho dos jogos. Para o autor, isso demonstra que a presença dos cães durante todo o processo foi um estímulo para a participação dos estudantes bem como para os benefícios que ocorreram; caso isso não acontecesse, é bem possível que a realização das atividades fosse comprometida. Consoante a essa opinião, um outro estudo realizado com estudantes e residentes de Medicina nos Estados Unidos resultou no aumento das emoções positivas em comparação aos estudantes que apenas interagiram com fotos de cães (Crossman et al., 2015). 
A partir das evidências obtidas e nos diversos benefícios descritos pela literatura sobre as intervenções assistidas por animais (Bert et al., 2016; Mandrá et al., 2019) se perpetua cada vez mais a ideia de implementação de programas de visitas animais ou animal visitation programs (AVP) para estudantes universitários. Com boa aceitação, alta eficiência e baixo custo, as AVPs podem promover estratégias para aliviar as adversidades promovidas pela vida acadêmica, sendo utilizadas em alta escala em outros países, diferentemente do Brasil que tem poucas unidades relatadas (Crossman \& Kazdin, 2015).

Como todo estudo, algumas limitações precisam ser abordadas. O tamanho da amostra é relativamente pequeno em comparação a outros estudos da área e também são necessárias pesquisas adicionais que incluam outros instrumentos de avaliação de bem-estar e saúde mental a fim de aprimorar os conhecimentos quanto a eficiência das IAAs sobre a vida dos estudantes universitários.

\section{Conclusão}

Conclui-se, diante dos resultados expostos, que o presente estudo promove subsídios a favor dos benefícios das interações com cães para estudantes universitários, por meio do efeito positivo sobre o bem-estar dado pelo maior número de emoções positivas relatadas, bem como sobre o pulso e pressão arterial sistólica, ambas reduzidas após a intervenção. Por conseguinte, faz-se necessário estudos a longo prazo com a avaliação da contribuição do cão no desempenho e diminuição do estresse do acadêmico bem como a necessidade de cães de assistência para casos específicos.

\section{Agradecimentos}

Os autores agradecem ao Ministério da Educação (MEC) pela concessão da bolsa de residência em área profissional da saúde - medicina veterinária, na área de concentração Pet Terapia: atividade, terapia e educação assistida por animais; ao Conselho Nacional de Desenvolvimento Científico e Tecnológico (CNPq) pela bolsa de produtividade em pesquisa (processo 308152/2019-0); a Universidade Federal de Pelotas e a Pró-Reitoria de Ensino por concessão de bolsas.

\section{Referências}

Andrews, F. M., \& Whitey, S. B. (1976). Social Indicators of well-being: America's perception of quality of life. Plenum Press.

Bert, F., Gualano, M. R., Camussi, E., Pieve, G., Voglino, G., \& Siliquini, R. (2016). Animal assisted intervention: A systematic review of benefits and risks. European Journal of Integrative Medicine, 8(5), 695-706.

Bjick, M. (2013). The Effects of a Therapy Animal on College Student Stress and Arousal. Master of Social Work Clinical Research Papers, 152. http://sophia.stkate.edu/msw_papers/152

Binfet, J. (2017). The Effects of Group-Administered Canine Therapy on University Students Wellbeing: A Randomized Controlled Trial. Anthrozoös, 30(3), 397-414.

Bradburn, N. M. (1969). The structure of psychological well-being. Aldine.

Crossman, M. K., \& Kazdin, A. E. (2015). Animal visitation programs in colleges and universities: An efficient model for reducing student stress. In A. H. Fine (Ed.), Handbook on animal-assisted therapy (pp. 333-337). Academic Press.

Crossman, M. K., Kazdin, A. E., \& Knudson, K. (2015). Brief unconstructed interaction with a dog reduces distress. Anthrozoös, 28(4), 649-659.

Diener, E. (1996). Subjective well-being in cross-cultural perspective. In G. Hector (Ed.), Key issues in cross-cultural psychology: selected papers from the Twelfth International Congress of the International Association for Cross-Cultural Psychology. Academic Press.

Delgado, C., Toukonen, M., \& Wheeler, C. (2018). Effect of Canine Play Interventions as a Stress Reduction Strategy in College Students. Nurse educator, 43(3), 149-153.

Estrela, C. (2018). Metodologia Científica: Ciência, Ensino, Pesquisa. Editora Artes Médicas.

Fernandez, A., Howse, E., Rubio-Valera, M., Thorncraft, K., Noone, J., Luu, X., Veness, B., Leech, M., Llewellyn, G., \& Salvador-Carulla, L. (2016). Settingbased interventions to promote mental health at the university: a systematic review. International Journal of Public Health, 61(7), 797-807.

Fine, A. H. (2010). Handbook on animal-assisted therapy: Theoretical foundations and guidelines for practice. Academic press. 
Fórum Nacional de Pró-reitores de Assuntos Comunitários e Estudantis (FONAPRACE) (2019). V pesquisa do perfil do socioeconômico e cultural dos(as) graduandos(as) das IFES - 2018 [Unpublished report]. http://www.andifes.org.br/wp-content/uploads/2019/05/V-Pesquisa-Nacional-de-PerfilSocioecon\%C3\%B4mico-e-Cultural-dos-as-Graduandos-as-das-IFES-2018.

Grajfoner, D., Harte, E., Potter, L. M., \& McGuigan, N. (2017). The Effect of Dog-Assisted Intervention on Student Well-Being, Mood, and Anxiety. International journal of environmental research and public health, 14(5), 483.

Graner, K. M., \& Ramos-Cerqueira, A. T. A. (2019). Revisão integrativa: Sofrimento psíquico em estudantes universitários e fatores associados. Ciência \& Saúde Coletiva, 24(4), 1327-1346.

Haggerty, J. M., \& Mueller, M. K. (2017). Animal-assisted Stress Reduction Programs in Higher Education. Innovative Higher Education, 42, 379-389.

Jegatheesan, B., Beetz, A., Choi, G., Dudzik, C., Fine, A., Garcia, R. M., Johnson, R.,Ormerod, E., Winkle, M., \& Yamazaki, K. (2014). White paper: The IAHAIO definitions for animal assisted intervention and guidelines for wellness of animals involved. [Unpublished report]. http://iahaio.org/wp/wpcontent/uploads/2021/01/iahaio-white-paper-2018-english.pdf

Kohlmann Jr, O., Guimarães, A. C., Carvalho, M. H. C., Chaves Jr, H.C., Machado, C.A., \& Praxedes, J.N. (1999). III Consenso Brasileiro de Hipertensão Arterial. Arquivos Brasileiros de Endocrinologia \& Metabologia, 43(4).

Lussi, I. A. O., Freitas, H. I. \& Pereira Junior, A. (2004). Proposta Teórica de um Instrumento de Auto-Avaliação em Saúde. Cadernos de Terapia Ocupacional da UFSCAR, 12(1), 5-14.

Mandrá, P. P., Moretti, T. C. D. F., Avezum, L. A., \& Kuroishi, R. C. S. (2019). Terapia assistida por animais: revisão sistemática da literatura. In CoDAS (Vol. 31, No. 3). Sociedade Brasileira de Fonoaudiologia.

Mcdowell, I. (2006). Measuring health: a guide to rating scales and questionnaires. Oxford University Press.

Nobre, M. O., Krug, F. D. M., Capella, S. O., Canielles, C., \& Pereira, C. S. (2017). Intervenções Assistidas por Animais: Uma nova perspectiva na educação. Revista Eletrônica de Veterinária, 18(2), 1-8.

Nogueira-Martins, L. A., \& Nogueira- Martins, M. C. F. (2018). Saúde mental e qualidade de vida de estudantes universitários. Revista Psicologia, Diversidade E Saúde, 7(3), 334-337.

Oliveira, J. A. (2006). Qualidade de vida e desempenho acadêmico de graduandos. Dissertação de doutorado, Universidade Estadual de Campinas, Repositório da Produção Científica e Intelectual da Unicamp.

Pendry, P., Carr, A. M., Roeter, S. M., \& Vandagriff, J. L. (2018). Experimental trial demonstrates effects of animal-assisted stress prevention program on college students' positive and negative emotion. Human-Animal Interaction Bulletin, 6(1), 81-97.

Sapin. C. F., Lima, C. M., Almeida, D. M., Ferraz, A., Albuquerque, M. S., Grill, J. D., Fripp, J. C, \& Nobre, M. O. (2020). Fisioterapia assistida por animais: o cão co-terapeuta como motivador e mediador dos exercícios para pacientes crônicos. Research, Society and Development, 9(11), e59591110214e59591110214.

Schleich, A. L. (2006). Integração na educação superior e satisfação acadêmica de estudantes ingressantes e concluintes. Tese de mestrado, Universidade Estadual de Campinas. Repositório da Produção Científica e Intelectual da Unicamp.

Silas, H. J., Binfet, J., \& Ford, A. T. (2019). Therapeutic for all? Observational assessments of therapy canine stress in an on-campus stress-reduction program. Journal of Veterinary Behavior, 32, 6-13.

Trammell, J. P. (2017). The Effect of Therapy Dogs on Exam Stress and Memory. Anthrozoös, 30(4), 607-621.

Williams, C., Emond, K., Maynord, K., Simpkins, J., Stumbo, A., \& Terhaar, T. (2018). An animal-assisted intervention's influence on graduate students' stress and anxiety prior to an examination. Open Access Library Journal, 5(9), 1-16.

Wood, E., Ohlsen, S., Thompson, J., Hulin, J., \& Knowles, L. (2018). The feasibility of brief dog-assisted therapy on university students stress levels: the PAwS study. Journal of Mental Health, 27(3), 263-268. 